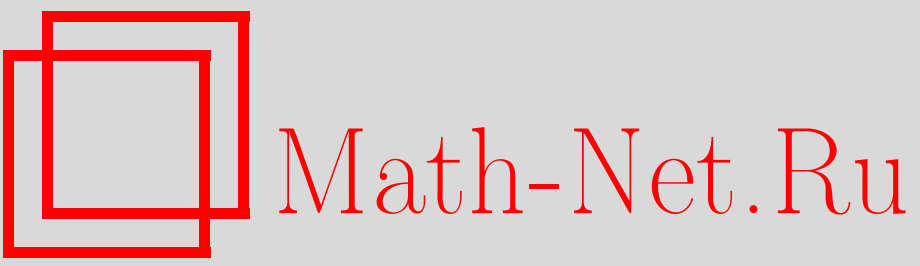

В. А. Скороходов, Д. О. Свиридкин, Регулярные периодические динамические ресурсные сети, Итоги науки и техн. Сер. Соврем. мат. и ее прил. Темат. обз., 2021, том 192, 117-124

DOI: https://doi.org/10.36535/0233-6723-2021-192-117-124

Использование Общероссийского математического портала Math-Net.Ru подразумевает, что вы прочитали и согласны с пользовательским соглашением

http: //www.mathnet.ru/rus/agreement

Параметры загрузки:

IP: 54.81 .137 .203

26 апреля 2023 г., 08:02:02 


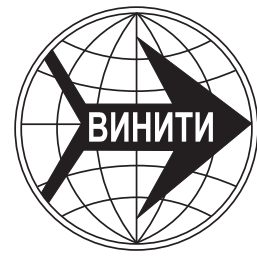

ИТОГИ НАУКИ И ТЕХНИКИ.

Современная математика и ее приложения.

Тематические обзоры.

Том 192 (2021). С. 117-124

DOI: $10.36535 / 0233-6723-2021-192-117-124$

УДК 519.1

\title{
РЕГУЛЯРНЫЕ ПЕРИОДИЧЕСКИЕ ДИНАМИЧЕСКИЕ РЕСУРСНЫЕ СЕТИ
}

\author{
(c) 2021 г. $\quad$ В. А. СКОРОХОДОВ, Д. О. СВИРИДКИН
}

\begin{abstract}
АннотАция. В работе изучается модель распределения ресурсного потока в динамической периодической ресурсной сети. Основной задачей является разработка методов нахождения предельного состояния (распределения) ресурса в динамической ресурсной сети. Показано, что для регулярных периодических динамических сетей предельное состояние существует и является единственным, а для его нахождения можно использовать подходы, разработанные для динамических сетей.
\end{abstract}

Ключевые слова: динамическая сеть, регулярная ресурсная сеть, ресурсный поток, распределение потока, пороговое значение, предельное состояние.

\section{REGULAR PERIODIC DYNAMIC RESOURCE NETWORKS}

\author{
(c) 2021 V. A. SKOROKHODOV, D. O. SVIRIDKIN
}

\begin{abstract}
A model of resource flow distribution in a dynamic periodic resource network is examined. Methods for finding the limiting state (distribution) of a resource in a dynamic resource network are developed. It is proved that for regular periodic dynamical networks, the limit state exists and is unique and can be found by methods developed for dynamic networks.
\end{abstract}

Keywords and phrases: dynamic network, regular resource network, resource flow, flow distribution, threshold value, limit state.

AMS Subject Classification: 05C21, 05C50, 90B10

1. Введение. Теория динамических потоков в сетях (теория динамических сетей), взяв свое начало еще в работах Л. Р. Форда и Д. Р. Фалкерсона (см., например, [17]), продолжила развитие в работах Д. Б. Орлина, Д. Е. Аронсона, М. В. Фоноберовой, Д. Д. Лозовану, Б. Клинца и др. (см. [13-16,18-21]). Динамическая сеть представляет собой ориентированную сеть, некоторые характеристики дуг которой (в частности, пропускные способности дуг) зависят от дискретного времени. Таким образом, поток в сети не является стационарным, а зависит от времени. В работах Я. М. Ерусалимского, В. А. Скороходова и М. В. Кузьминовой (см. $[2,7,10])$ описан общий подход к решению потоковых задач в динамических сетях, который состоит в построении вспомогательной «статической» сети, описывающей динамику изменения исходной сети, и сведении потоковой задачи в исходной динамической сети к аналогичной задаче на вспомогательной.

Немного в стороне от классической теории потоков в сетях стоят ресурсные сети, введенные и довольно хорошо изученные О. П. Кузнецовым и Л. Ю. Жиляковой (см. [3-6, 19]). Ресурсная сеть - это сеть без источников, для каждой дуги которой указана пропускная способность, а для каждой вершины - величина находящегося в ней ресурса. В каждый момент дискретного времени ресурс каждой вершины перераспределяется между смежными с ней вершинами по определенным правилам. Таким образом, между каждыми последовательными моментами времени по дугам сети проходит поток. При этом правила функционирования сети таковы, что обязательно 
выполняются два условия. Первое - это условие замкнутости сети, т.е. ресурс ни в какой вершине сети не добавляется извне и не исчезает. Второе - условие неразрывности: ресурс, выходящий из вершины, вычитается из ее ресурса, а входящий в вершину прибавляется к ее ресурсу.

В настоящей работе изучается модель распределения ресурсного потока в динамической периодической ресурсной сети. Основной задачей является разработка методов нахождения предельного состояния (распределения) ресурса в динамической ресурсной сети. Показано, что для регулярных динамических сетей предельное состояние существует и является единственным, а для его нахождения можно использовать подходы, разработанные для динамических сетей в $[2,7,10]$.

2. Основные понятия. Напомним основные определения и утверждения (см. $[2-7,10,19])$.

Определение 1. Ресурсной сетью называют связную ориентированную сеть $G(X, E)$ (где $\left.X=\left\{x_{1}, \ldots, x_{n}\right\}\right)$ без стоков, для каждой дуги $\left(x_{i}, x_{j}\right)$ которой указана пропускная способность $r_{i j}$, и задана вектор-функция $Q(t)=\left(q_{1}(t), \ldots, q_{n}(t)\right)$, где $q_{i}(t) \geqslant 0$ для всех $i \in[1 ; n]_{\mathbb{Z}}$.

Величина $q_{i}(t)$ называется количеством ресурса в вершине $x_{i}$ в момент времени $t$.

Для того чтобы определить вектор-функцию $Q(t)$, задается вектор $Q(0)$ начального распределения ресурса в сети $G$ и указываются правила перераспределения ресурсов (правила функционирования сети):

$$
q_{i}(t+1)=q_{i}(t)-\sum_{j=1}^{n} F_{i j}(t)+\sum_{j=1}^{n} F_{j i}(t) \quad \forall i \in[1 ; n]_{\mathbb{Z}},
$$

где $F_{i j}(t)$ - величина ресурсного потока, выходящего по дуге $(i, j)$ в момент времени $t$, определяется следующим образом:

$$
F_{i j}(t)= \begin{cases}r_{i j}, & q_{i}>\sum_{k=1}^{n} r_{i k} ; \\ \frac{r_{i j}}{\sum_{k=1}^{n} r_{i k}} \cdot q_{i}(t), & q_{i} \leqslant \sum_{k=1}^{n} r_{i k} .\end{cases}
$$

Величину суммарного ресурса сети обозначим через $W$, т.е.

$$
W=\sum_{i=1}^{n} q_{i}(0)
$$

Определение 2. Состояние $Q(t)$ называется устойчивым, если выполняется $Q(t)=Q(t+1)$.

Согласно правилам перераспределения ресурса, если $Q(t)$ устойчиво, то для всех натуральных $i$ имеет место равенство $Q(t)=Q(t+i)$.

Определение 3. Состояние $Q^{*}=\left(q_{1}^{*}, \ldots, q_{n}^{*}\right)$ называется асимптотически достижимым из состояния $Q(0)$, если для каждого $i \in[1 ; n]_{\mathbb{Z}}$ и всякого $\varepsilon>0$ существует $t_{\varepsilon}$ такое, что для всех $t>t_{\varepsilon}$ имеет место неравенство $\left|q^{*}-q_{i}(t)\right|<\varepsilon$.

Определение 4. Состояние $Q^{*}$ называется предельным, если оно либо устойчиво и существует такой момент времени $t$, что $Q^{*}=Q(t)$, либо оно асимптотически достижимо из состояния $Q(0)$.

Определение 5. Ресурсную сеть будем называть эргодической, если она является сильно связной.

Определение 6. Эргодическую ресурсную сеть будем называть регулярной, если существует по крайней мере два цикла, длины которых являются взаимно простыми числами. 
3. Периодические динамические сети. Пусть $G(X, E, r, D)$-регулярная периодическая динамическая ресурсная сеть, т.е. такая сеть, для каждой дуги $u$ которой в каждый момент времени $t$ указана величина $r_{i j}(t)$ - пропускная способность дуги $\left(x_{i}, x_{j}\right)$ в момент $t$ и имеет место соотношение $r_{i j}(t)=r_{i j}(t+D)$. При этом полагаем, что время является дискретным и пропускные способности дуг сети $G$ не обращаются в ноль. Рассмотрим вопрос о существовании и единственности предельного состояния для таких сетей.

В [9] изучена задача нахождения порогового значения в таких сетях. Для этих целей процесс перераспределения ресурсов в динамической ресурсной сети моделировался при помощи вспомогательной ресурсной сети большего размера, но на которой пропускные способности дуг не зависят от времени (см. также $[7,10])$. Показано, что, несмотря на регулярность исходной сети, вспомогательная сеть обязательно является $D$-циклической, что существенно влияет на существование единственного предельного состояния в случае малого ресурса.

Отметим, что поскольку для каждой дуги динамической ресурсной сети указывается периодическая зависимость пропускной способности от времени, значит, фактически для такой сети задана периодическая последовательность матриц пропускных способностей $R(t)$.

Для дальнейшего изложения введем в рассмотрение следующие обозначения. $c(t)$ - вектор суммарных пропускных способностей вершин в момент времени $t$, т.е.

$$
c_{i}(t)=\sum_{j=1}^{n} r_{i j}(t),
$$

$P(t)$ - стохастическая матрица, определяющая долевое распределение ресурса по дугам сети (см. [6]) в момент времени $t$.

Для введенных величин основное правило функционирования ресурсной сети (1) для динамических ресурсных сетей можно записать в виде

$$
Q(t+1)=\max \{Q(t)-c(t), 0\}+\min \{Q(t), c(t)\} \cdot P(t) .
$$

В соотношении (2) минимум и максимум берутся поэлементно, т.е. если для некоторых векторов $\boldsymbol{x}$ и $\boldsymbol{y}$ вектор $\boldsymbol{z}=\max \{\boldsymbol{x}, \boldsymbol{y}\}$, то это означает, что $z_{i}=\max \left\{x_{i}, y_{i}\right\}$ для всех $i \in[1 ; n]_{\mathbb{Z}}$. Таким образом, каждая вершина $x_{i} i \in[1 ; n]_{\mathbb{Z}}$ в момент $t$ отдает не более $c_{i}(t)$ ресурса, который распределяется пропорционально долям, определяемых матрицей $P(t)$.

Далее для удобства правило функционирования (2) будем иногда записывать в сокращенной форме:

$$
Q(t+1)=\mathcal{A}_{t}(Q(t))
$$

Отметим, что каждой паре $(c, P)$ можно однозначно поставить в соответствие ориентированную сеть $G\left(X, E_{P}\right)$, на которой пропускная способность каждой дуги $\left(x_{i}, x_{j}\right)$ определяется следующим правилом: $r_{i j}=P_{i j} \cdot c_{i}$.

Лемма 1. Пусть $P_{1}$ и $P_{2}$ - регулярные стохастические матрицы одинакового порядка и вектор с $=(1, \ldots, 1)$, и пусть $G\left(X, E_{1}\right)$ и $G\left(X, E_{2}\right)$ - графы, соответствуюшие парам $\left(c, P_{1}\right)$ u $\left(c, P_{2}\right)$ соответственно. Для того чтобы матрича $P \cdot Q$ была регулярной стохастической матрицей, достаточно, чтобы $E_{1} \subseteq E_{2}$ или $E_{2} \subseteq E_{1}$.

Доказательство. Не нарушая общности, полагаем $E_{1} \subseteq E_{2}$ и $E_{0}=E_{2} \backslash E_{1}$. Также можно считать, что множества дуг $E_{1}$ и $E_{2}$ являются бинарными отношениями, определенными на множестве вершин $X$.

Рассмотрим граф $G\left(X, E_{3}\right)$, соответствующий произведению матриц $P_{1} \cdot P_{2}$. Множество дуг этого графа получить как композицию отношений $E_{1} \circ E_{2}$ (см. [8]). Тогда

$$
E_{1} \circ E_{2}=E_{1} \circ\left(E_{1} \cup E_{0}\right)=E_{1}^{2} \cup\left(E_{1} \circ E_{2}\right) .
$$

Поскольку матрица $P_{1}^{2}$ является регулярной как степень регулярной матрицы (см. [1]), следовательно, соответствующий ей граф $G\left(X, E_{1}^{2}\right)$ содержит по крайней мере два цикла, длины которых взаимно просты (см. [11]). Отметим также, что граф $G\left(X, E_{1}^{2}\right)$ является частичным графом графа $G\left(X, E_{3}\right)$, а значит, и $G\left(X, E_{3}\right)$ содержит по крайней мере два цикла, длины которых 
взаимно простые. А поскольку $G\left(X, E_{3}\right)$ соответствует матрице $P_{1} \cdot P_{2}$, следовательно, последняя является регулярной.

Следует отметить, что произведение регулярных матриц может и не быть регулярной матрицей. Покажем эту ситуацию на следующем примере.

Пример 1. Рассмотрим следующие регулярные матрицы

$$
A=\left(\begin{array}{cccc}
0,5 & 0,5 & 0 & 0 \\
0 & 0 & 0 & 1 \\
1 & 0 & 0 & 0 \\
0 & 0 & 1 & 0
\end{array}\right), \quad B=\left(\begin{array}{cccc}
0 & 1 & 0 & 0 \\
0 & 0 & 1 & 0 \\
0,5 & 0 & 0 & 0,5 \\
0 & 0 & 1 & 0
\end{array}\right)
$$

На рис. 1 представлены графы $G_{A}$ и $G_{B}$, соответствующие матрицам $A$ и $B$.
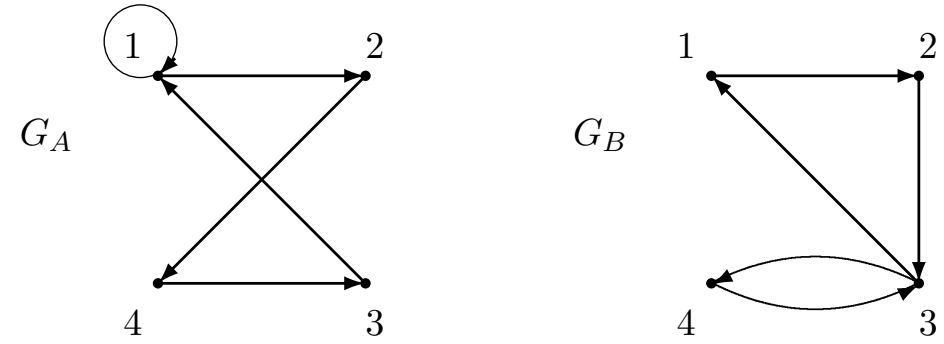

Рис. 1. Графы $G_{A}$ и $G_{B}$.

Произведения рассматриваемых матриц имеют вид

$$
A \cdot B=\left(\begin{array}{cccc}
0 & 0,5 & 0,5 & 0 \\
0 & 0 & 1 & 0 \\
0 & 1 & 0 & 0 \\
0,5 & 0 & 0 & 0,5
\end{array}\right), \quad B \cdot A=\left(\begin{array}{cccc}
0 & 0 & 0 & 1 \\
1 & 0 & 0 & 0 \\
0,25 & 0,25 & 0,5 & 0 \\
1 & 0 & 0 & 0
\end{array}\right) .
$$

На рис. 2 представлены графы $G_{A}$ и $G_{B}$, соответствующие матрицам $A$ и $B$.
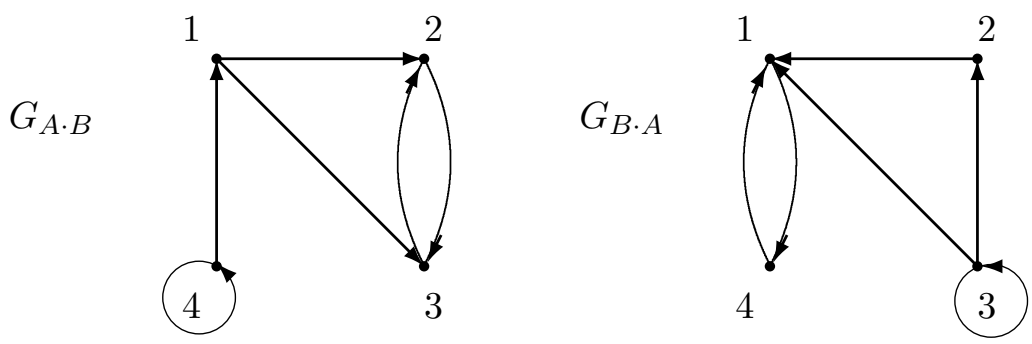

Рис. 2. Графы $G_{A \cdot B}$ и $G_{B \cdot A}$.

Обе матрицы (и $A \cdot B$, и $B \cdot A)$ не являются регулярными, поскольку

1. соответствующие им графы не являются сильно связными,

2. в отдельных компонентах сильной связности только по одному простому циклу длины 2.

Для динамической сети рассмотрим ее временную развертку (см. $[9,10])$ - ориентированный граф $G^{\prime}\left(X^{\prime}, E^{\prime}\right)$, такой, что для каждой вершины $x \in X$ графа $G$ ставится в соответствие $D$ вершин $A_{x}=\left\{x^{0}, \ldots, x^{D-1}\right\}$ на развертке $G^{\prime}$, для каждой дуги $u \in E$ (положим для определенности $u=(x, y))$ исходного графа $G$ ставится в соответствие $D$ дуг $\left\{u_{0}, \ldots, u_{D-1}\right\}$ на развертке $G^{\prime}$ так, что $u_{i}=\left(x^{i}, y^{(i+1)} \bmod D\right)$ для всех $i \in[0 ; D-1]_{\mathbb{Z}}$. Вес дуги $u_{i} \in E^{\prime}$ полагается равным весу дуги $u \in E$ в момент времени $i$. 
Замечание. На развертке $G^{\prime}$ множество вершин разбивается на непересекающиеся множества $V_{i}=\left\{x_{1}^{i}, \ldots, x_{n}^{i}\right\}$, которые мы будем называть $i$-м временным слоем.

Тогда при введении сплошной нумерации вершин матрица долевого распределения ресурса для развертки $G^{\prime}$ будет иметь следующий вид:

$$
P_{G^{\prime}}=\left(\begin{array}{ccccc}
\Theta & P(0) & \Theta & \ldots & \Theta \\
\Theta & \Theta & P(1) & \ldots & \Theta \\
\vdots & \vdots & \vdots & \ddots & \vdots \\
\Theta & \Theta & \Theta & \ldots & P(D-2) \\
P(D-1) & \Theta & \Theta & \ldots & \Theta
\end{array}\right)
$$

где $\Theta$ - квадратная матрица, состоящая из нулей. Отметим, что матрицу $P_{G^{\prime}}$ можно также записать в виде $P_{G^{\prime}}=P \cdot S^{n}$, где $P=\operatorname{diag}\{P(0), P(1), \ldots, P(D-1)\}$ - блочно-диагональная матрица, a $S$ - матрица оператора циклического сдвига вправо.

Таким образом, как и в [9], процесс перераспределения ресурса между вершинами динамической сети будем моделировать аналогичным процессом на развертке, при этом функционирование вспомогательной ресурсной сети описывается схожим с (2) соотношением

$$
Q^{\prime}(t+1)=\max \left\{Q^{\prime}(t)-C, 0\right\} \cdot S^{n}+\min \left\{Q^{\prime}(t), C\right\} \cdot P_{G^{\prime}},
$$

здесь $C=(c(0), c(1), \ldots, c(D-1))$. Начальное состояние для развертки задается следующим образом $Q^{\prime}(0)=(Q(0), Q(1), \ldots, Q(D-1))$.

Единственным отличием от функционирования ресурсных сетей с постоянными пропускными способностями является наличие оператора $S^{N}$, отвечающего за перенос «избыточного» ресурса в вершинах на следующий временной слой в те же самые вершины.

4. Малые ресурсы и предельное состояние. Рассмотрим вопрос о существовании единственного предельного состояния в случае малого ресурса, т.е. когда $W \leqslant T$.

Построим $D$ сетей с постоянными пропускными способностями следующим образом.

Сеть $G_{i}(i=0, \ldots, D-1)$ определяется парой $(c(i), B(i))$, где $B(i)=P(i) \cdot P(i+1) \cdot \ldots \cdot P(D-$ 1) $\cdot P(0) \cdot \ldots \cdot P(i-1)$.

Каждая из этих сетей является регулярной, поскольку по лемме 1 каждая матрица $B(i)$, определяющая долевое распределение потока в сети $G_{i}$, является регулярной. В регулярных сетях при малых ресурсах существует единственное предельное распределение (см. [4]).

Лемма 2. Пусть $Q_{i}^{*}$ - вектор предельного состояния при $W=1$ в сети $G_{i}$. Тогда

$$
Q_{i+1(\bmod D)}^{*}=Q_{i}^{*} \cdot P(i) .
$$

Доказательство. Рассмотрим для сети $G_{i+1(\bmod D)}$ в качестве начального состояния вектор $Q_{* i}(0)=Q_{i}^{*} \cdot P(i)$. Тогда поскольку рассматривается случай малого ресурса, значит, $Q_{* i}(1)=$ $\mathcal{A}\left(Q_{* i}(0)\right)=\left(Q_{i}^{*} \cdot P(i)\right) \cdot B(i+1(\bmod D))$. Пользуясь определением $B(i)$, преобразуем к следующему виду:

$$
Q_{* i}(1)=\left(Q_{i}^{*} \cdot P(i)\right) \cdot P(i+1(\bmod D)) \cdot \ldots \cdot P(D-1) \cdot P(0) \cdot \ldots \cdot P(i-1) \cdot P(i) .
$$

Перегруппируем множители и получим $Q_{* i}(1)=Q_{i}^{*} \cdot B(i) \cdot P(i)$.

Поскольку вектор $Q_{i}^{*}$ является предельным состоянием для сети $G_{i}$, тогда $Q_{i}^{*} \cdot B(i)=Q_{i}^{*}$. Таким образом, получили

$$
Q_{* i}(1)=Q_{i}^{*} \cdot P(i)=Q_{* i}(0) .
$$

Последнее означает, что состояние $Q_{* i}(0)=Q_{i}^{*} \cdot P(i)$ является устойчивым, а значит, предельным для сети $G_{i+1(\bmod D)}$. Таким образом, в силу единственности предельного состояния имеет место равенство (4).

Теорема 1. Вектор $Q_{* 1}^{\prime}=\left(Q_{0}^{*}, \ldots, Q_{D-1}^{*}\right)$ является предельным состоянием для ресурсной сети $G^{\prime}$ при $W=1$. 
Доказательство. Действительно, если рассмотреть вектор $Q^{\prime}(0)=Q_{* 1}^{\prime}$ в качестве начального вектора для вспомогательной ресурсной сети $G^{\prime}$, то следующее состояние $Q^{\prime}(1)=\mathcal{A}^{\prime}\left(Q^{\prime}(0)\right)=$ $Q_{* 1}^{\prime} \cdot P_{G^{\prime}}$, поскольку $W=1$. Однако

$$
Q_{* 1}^{\prime} \cdot P_{G^{\prime}}=\left(Q_{D-1}^{*} \cdot P(D-1), Q_{0}^{*} \cdot P(0), \ldots, Q_{D-2}^{*} \cdot P(D-2)\right),
$$

по лемме 2 это равно $\left(Q_{0}^{*}, \ldots, Q_{D-1}^{*}\right)=Q_{* 1}^{\prime}=Q^{\prime}(0)$, т.е. состояние $Q_{* 1}^{\prime}$ является устойчивым для сети $G^{\prime}$, а значит, предельным.

Замечание. В данной работе мы рассматриваем сильно регулярные динамические периодические ресурсные сети, т.е. такие сети, для которых все матрицы $B(i)$ являются регулярными. Однако для существования единственного предельного состояния на вспомогательной сети $G^{\prime}$ при $W=1$ достаточно и более слабого условия: «хотя бы одна из матриц $B(i)$ должна быть регулярной».

Теорема 2. В сети $G^{\prime}$ при $W \leqslant T$ существует единственное предельное состояние вида

$$
Q^{\prime *}=\left(W \cdot Q_{0}^{*}, W \cdot Q_{1}^{*}, \ldots, W \cdot Q_{D-1}^{*}\right) .
$$

Доказательство. Рассмотрим состояние сети $G^{\prime}$ в момент $t: Q^{\prime}(t)=Q^{*}+\Delta(t)$. Отметим, что сумма всех компонент каждого вектора $\Delta(t)$ равна нулю, поскольку суммарная величина ресурса в сети не меняется с течением времени.

Рассмотрим процесс функционирования сети $G^{\prime}$. Подставляя $Q^{\prime}(t)$ в $(3)$, имеем

$$
Q^{\prime *}+\Delta(t+1)=\max \left(Q^{\prime *}+\Delta(t)-C, 0\right) \cdot S^{n}+\min \left(Q^{\prime *}+\Delta(t), C\right) \cdot P_{G^{\prime}} .
$$

Группируя и вынося $Q^{\prime *}$ из минимума по правилу $\min (a+b, a+c)=a+\min (b, c)$, получим

$$
Q^{\prime *}+\Delta(t+1)=\max \left(\Delta(t)-\left(C-Q^{*}\right), 0\right) \cdot S^{n}+Q^{\prime *}+\min \left(\Delta(t), C-Q^{*}\right) \cdot P_{G^{\prime}} .
$$

Учитывая, что $C \geqslant Q^{*}$, обозначим разность $C-Q^{*}$ через $C^{\prime}$ и получим, что изменение значений $\Delta(t)$ описывается соотношением (5), аналогичным (3), но с другими пропускными способностями вершин:

$$
\Delta(t+1)=\max \left(\Delta(t)-C^{\prime}, 0\right) \cdot S^{n}+\min \left(\Delta(t), C^{\prime}\right) \cdot P_{G^{\prime}} .
$$

Докажем, что $\lim _{t \rightarrow \infty} \Delta(t)=0$.

Представим вектор $\Delta(t)$ в виде суммы $\Delta(t)=\Delta^{+}(t)+\Delta^{-}(t)$ - по положительной и отрицательной частям $\Delta(t)$. Таким образом, $\Delta^{+}(t) \geqslant 0$ и $\Delta^{-}(t) \leqslant 0$. Тогда соотношение (5) примет следующий вид:

$$
\Delta(t+1)=\max \left(\Delta^{+}(t)-C^{\prime}, 0\right) \cdot S^{n}+\min \left(\Delta^{+}(t), C^{\prime}\right) \cdot P_{G^{\prime}}+\Delta^{-}(t) \cdot P_{G^{\prime}} .
$$

Отметим, что сумма компонент вектора $\Delta^{-}(t) \cdot P_{G^{\prime}}$ совпадает с суммой компонент $\Delta^{-}(t)$ и отрицательна. Сумму оставшихся положительных векторов обозначим через $\hat{\Delta}^{+}(n)$.

Нам осталось показать, что через некоторое конечное время $\tau$ найдется такая вершина $x \in G^{\prime}$, что $\left(\Delta^{-}(t+\tau) \cdot P_{G^{\prime}}\right)_{x} \neq 0$ и $\left(\hat{\Delta}^{+}(t+\tau)\right)_{x} \neq 0$.

Предположим, что это не так, что для любого промежутка времени для каждой вершины $x$ либо $\left(\Delta^{-}(t+\tau) \cdot P_{G^{\prime}}\right)_{x}=0$, либо $\left(\hat{\Delta}^{+}(t+\tau)\right)_{x}=0$. Отсюда следует, что имеет место следующее соотношение

$$
\Delta^{-}(t+1)=\Delta^{-}(t) \cdot P_{G^{\prime}} .
$$

Таким образом, изменение значений вектор-функции $\Delta^{-}(t)$ описывается марковской цепью, которая определяется стохастической матрицей ресурсной сети $G^{\prime}$. Такая ситуация соответствует условиям теоремы 1 для единичного ресурса в сети $G^{\prime}$. Это означает, что существует предельное «состояние» $\Delta_{*}^{-}=\delta \cdot\left(Q_{0}^{*}, \ldots, Q_{D-1}^{*}\right)$, где

$$
\delta=\sum_{x \in G^{\prime}} \Delta^{-}(0),
$$

при этом вектор $\Delta_{*}^{-}$не имеет нулевых компонент. Следовательно, существует конечное число $\theta$ (более того, можно показать, что для регулярных сетей $\theta \leqslant n)$ такое, что вектор $\Delta^{-}(t+\theta)=$ $\Delta^{-}(t) \cdot\left(P_{G^{\prime}}\right)^{\theta}$ не содержит нулевых компонент. Получили противоречие. 
Таким образом, через каждые $n$ итераций найдется такая вершина $x \in G^{\prime}$, что

$$
\left(\Delta^{-}(t+n) \cdot P_{G^{\prime}}\right)_{x} \neq 0, \quad\left(\hat{\Delta}_{x}^{+}(t+n)\right)_{x} \neq 0 .
$$

Следовательно, сумма отрицательных компонент вектор-функции $\Delta(t)$ уменьшается через каждые $n$ итераций. Сумма положительных компонент по абсолютному значению совпадает с суммой отрицательных. Таким образом, общая сумма модулей компонент вектор-функции $\Delta$ стремится к нулю. Последнее означает, что $\lim _{t \rightarrow \infty} \Delta(t)=0$.

Таким образом, поскольку процесс перераспределения ресурсов в сети $G^{\prime}$ моделирует аналогичный процесс в исходной динамической сети $G$ (см. [9]), значит, последовательность $Q^{\prime *}=$ $\left(W \cdot Q_{0}^{*}, W \cdot Q_{1}^{*}, \ldots W \cdot Q_{D-1}^{*}\right)$ образует набор предельных состояний (см. [4]) в исходной сети $G$.

5. Большие ресурсы и единственность предельного состояния. Теперь рассмотрим вопрос о существовании единственного предельного состояния в случае большого ресурса, т.е. когда $W>T$.

Теорема 3. Для любой регулярной периодической динамической ресурсной сети $G$, для любого начального состояния при $W>T$ существует предельное состояние.

Доказательство. Рассмотрим предельное состояние $Q^{*}$ для вспомогательной сети при $W=T$. Построим вспомогательную сеть $G^{\prime \prime}$, соответствующую паре $\left(C^{\prime \prime}, P^{\prime \prime}\right)$, где $C^{\prime \prime}=C-Q^{\prime *}$, а матрица $P^{\prime \prime}$ получена из матрицы $P_{G^{\prime}}$ таким образом: если $C_{i}^{\prime \prime}=0$, то $P_{i}^{\prime \prime}=\left(P_{G^{\prime}}\right)_{i}$. В противном случае элементы $i$-й строки $P^{\prime \prime}$ определяется соотношением

$$
P_{i j}^{\prime \prime}= \begin{cases}1, & j=i+n(\bmod D), \\ 0, & j \neq i+n(\bmod D),\end{cases}
$$

при этом величина $C_{i}^{\prime \prime}$ полагается равной $W-T$.

Сеть $G^{\prime \prime}$ получена из $G^{\prime}$ удалением насыщенных ресурсным потоком дуг, а в случае, если после такого удаления из какой-то вершины не осталось выходящих дуг, то достраивается дуга, ведущая в вершину следующего слоя. Последняя дуга означает перенос «оставшегося» ресурса в вершине исходной динамической ресурсной сети. Таким образом, сеть $G^{\prime \prime}$ моделирует распределение «оставшегося» ресурса величины $W-T$, т.е. величины превышения порогового значения.

Отметим, что сеть $G^{\prime \prime}$ разбивается на полуэргодические компоненты связности, состоящие из невозвратных вершин и изолированных (см. [11]) эргодических компонент, при этом, если изолированная компонента содержит хотя бы одну вершину множества $A_{x}$, то она содержит все вершины этого множества. Величина ресурса невозвратных вершин со временем станет равной нулю, и весь оставшийся «нераспределенным» ресурс соберется в изолированных эргодических компонентах. Таким образом, будем рассматривать ресурсный поток только на изолированных эргодических компонентах.

Для каждой такой изолированной компоненты имеют место следующие факты:

1. если она является простым циклом, т.е. содержит вершины только одного множества $A_{x}$ и только достроенные дуги, обеспечивающие перенос ресурса на следующий временной слой, то предельное состояние в такой компоненте является единственным;

2. если ее суммарный ресурс равен нулю, то ее предельное состояние является единственным (нулевым);

3. если она не является простым циклом и ее суммарный ресурс не равен нулю, то найдем для нее пороговое значение (см. $[9,12])$ и повторим действие, описанное для вспомогательной сети $G^{\prime}$. Структура вспомогательной сети $G^{\prime}$ такова, что, повторяя такой процесс для оставшегося ресурса, в итоге будут оставаться только простые циклы, проходящие по всем временным слоям.

Количество таких построений не превышает числа вершин исходной сети. Таким образом, предельное состояние ресурсной сети $G^{\prime}$ может быть получено суммированием соответствующих компонент предельных состояний всех полученных в итоге вспомогательных сетей. 
Подход, примененный в доказательстве, позволяет говорить о существовании предельного состояния в случае $W>T$, но не позволяет определять его. Предельное состояние в случае больших ресурсов, так же, как и для не динамических ресурсных сетей, зависит от начального состояния. Для его определения необходимо знать, в каких пропорциях распределится «остаток» ресурса по изолированным эргодическим компонентам.

\section{СПИСОК ЛИТЕРАТУРЫ}

1. Гантмахер Ф. Р. Теория матриц. - М.: Физматлит, 2010.

2. Ерусалимский Я. М., Скороходов В. А., Кузъминова М. В., Петросян А. Г. Графы с нестандартной достижимостью: задачи, приложения. - Ростов-на-Дону: ЮФУ, 2009.

3. Жилякова Л. Ю. Несимметричные ресурсные сети. І. Процессы стабилизации при малых ресурсах// Автомат. телемех. - 2011. - 4. - С. 133-143.

4. Жилякова Л. Ю. Эргодические циклические ресурсные сети. І. Колебания и равновесные состояния при малых ресурсах// Управл. бол. сист. - 2013. - 43. - С. 34-54.

5. Жилякова Л. Ю. Эргодические циклические ресурсные сети. IІ. Большие ресурсы// Управл. бол. сист. - 2013. - 45. - С. 6-29.

6. Кузнецов О. П., Жилякова Л. Ю. Двусторонние ресурсные сети - новая потоковая модель// Докл. PAH. - 2010. - 433, № 5. - С. 609-612.

7. Кузъминова М. В. Периодические динамические графы. Задача о максимальном потоке// Изв. вузов. Сев.-Кав. рег. Естеств. науки. - 2008. - 5. - С. 16-20.

8. Новиков Ф. А. Дискретная математика. - СПб.: Питер, 2013.

9. Скороходов B. А., Абдулрахман X. Динамические ресурсные сети. Случай малого ресурса// Вестн. ВГУ. Физ. Мат. - 2018. - 4. - С. 186-194.

10. Скороходов B. А. Потоки в сетях с меняющейся длительностью прохождения// Изв. вузов. Сев.-Кав. рег. Естеств. науки. - 2011. - 1. - С. 21-26.

11. Скороходов B. A. Устойчивость и стационарное распределение на графах с нестандартной достижимостью// Изв. вузов. Сев.-Кав. рег. Естеств. науки. - 2007. - 4. - С. 17-21.

12. Скороходов B. А. Задача нахождения порогового значения в эргодической ресурсной сети, 2016.

13. Aronson J. E. A survey of dynamic network flows// Ann. Oper. Res. - 1989. — 20. - P. 1-66.

14. Erzin A. I., Takhonov I. I. The problem of finding of balanced flow// J. Appl. Industr. Math. — 2005. 8, № 3 (23). - P. 58-68.

15. Fonoberova M., Lozovanu D. The maximum flow in dynamic networks// Comput. Sci. J. Moldova. - 2004. - 3, № 36. - P. 387-396.

16. Fonoberova M., Lozovanu D. The minimum cost multicommodity flow problem in dynamic networks and an algorithm for its solving// Comput. Sci. J. Moldova. - 2005. - 1, № 37. - P. 29-36.

17. Ford L. R., Fulkerson D. R. Constructing maximal dynamic flows from static flows// Oper. Res. - 1958. - 6. - P. 419-433.

18. Klinz B. Woeginger C. One, two, three, many, or: complexity aspects of dynamic network flows with dedicated arcs// Oper. Res. Lett. — 1998. - 22. — P. 119-127.

19. Kuznetsov O. P. Nonsymmetric resource networks. The study of limit states// Manag. Prod. Eng. Rev. 2011. - 2, № 3. - P. 33-39.

20. Orlin J. B. Maximum-throughput dynamic network flows// Math. Progr. — 1983. — 27. — P. $214-231$.

21. Skorokhodov V. A., Chebotareva A. S. The Maximum Flow Problem in a Network with Special Conditions of Flow Distribution// J. Appl. Industr. Math. — 2015. — 9, № 3. — P. 435-446.

Скороходов Владимир Александрович

Южный федеральный университет, Ростов-на-Дону

E-mail: vaskorohodov@sfedu.ru

Свиридкин Дмитрий Олегович

Южный федеральный университет, Ростов-на-Дону

E-mail: sv.11@mail.ru 